\title{
Recent Developments in Cancer Treatment: A Review
}

Maria Toloudi', Panagiotis Apostolou', Marina Chatziioannou', Eleni Kourtidou', loanna Vlachou', Georgia Mimikakou', Aikaterini Chlichlia² and loannis Papasotiriou ${ }^{1 *}$

${ }^{1}$ Research Genetic Cancer Centre Ltd (R.G.C.C. Ltd), Greece

${ }^{2}$ Department of Molecular Biology and Genetics, Democritus University of Thrace, Greece

\begin{abstract}
According to the latest cancer statistics presented worldwide, there has been a dramatic increase in the rates of occurrence of some cancers, particularly in the more developed countries. Although many therapeutic strategies to prevent and/or cure this disease have been proposed and evaluated by clinicians and researchers, there remains a need to find more effective approaches. Side effects such as toxicity and drug resistance are two of the most frequent problems faced during chemotherapy.
\end{abstract}

Small - molecule drugs are being intensively pursued as new anticancer therapeutics. Oncology drug discovery has benefited significantly from progress in understanding how to target kinases with small molecules that were found to be correlated with the disease. One reason for this is that many kinases have been found to be intimately involved in the processes leading to tumor cell proliferation and survival. Monoclonal antibodies, that are produced in vitro, can be used in cancer treatment in a number of ways. They may enhance the immune system by reacting with certain types of cancer cells. They can be programmed to act against specific cell growth factors to interfere with the growth of cancer cells. Furthermore, they may be linked to anticancer drugs, radioactive substances, other biologic therapies, or other toxins (antibody - drug conjugates). Finally, the usage of cytotoxic monoclonal antibodies during the process of bone marrow transplantation may be a key to improve the efficacy of the method.

The objective of this review was to present some of the new cancer treatment modalities that have been developed. The advantages of each method including its safety and efficacy have been highlighted. The present study may support the improvement and development of new therapeutic approaches.

Keywords: Cancer therapy; ADCs; Monoclonal antibodies; Drug toxicity

\begin{abstract}
Abbreviations: ADC: Antibody Drug Conjugate; SMDs: Small Molecule Drugs; moabs: Monoclonal Antibodies; PIs: Proteasome Inhibitors; CDK: Cyclin-dependent Kinase; EGFR: Epidermal Growth Factor Receptor; GCC: Guanyl Cyclase C; MMAE: Monomethyl Auristatin E; ALL: Acute Lymphoblastic Leukemia
\end{abstract}

\section{Introduction}

Cancer was first described by Hippocrates using the Greek word "karkinos" (crab or crayfish) [1]. This name came from the appearance of the cut surface of a solid malignant tumor, with "the veins stretched on all sides as the crab has its feet, whence it derives its name" [2]. According to the World Health Organization, it is estimated that worldwide there will be almost 22.2 million new cancer cases diagnosed annually by 2030 . The overall survival times of patients with many types of malignancies remain low. The above findings underline the urgent need for the development of new therapeutic models, and the improvement of the existing ones, to inhibit cancer progression and deliver improved therapeutic outcomes.

\section{Targeted Therapeutic Approach}

The targeted or molecularly targeted therapeutic concept involves the use of a medicinal compound to block and/or minimize the growth of cancer cells. During this process the normal tissues are not affected, and this kind of treatment may be more effective and less cytotoxic than other non-targeted traditional chemotherapies. Consequently, the primary goal of targeted therapies is to fight cancer cells with greater precision and potentially fewer side effects. Small-molecule drugs (SMDs), monoclonal antibodies (moabs), antibody drug conjugates (ADCs) as well as cancer vaccines are some of the basic classified agents that are broadly used.

\section{SMDs}

This kind of medication belongs to the class of cutting-edge anti-cancer drugs. SMDs can penetrate the cell membrane and the blood-brain barrier more easily. They are simple organic molecules with a low molecular weight $(<1000 \mathrm{kDa})$ that can be delivered orally or intravenously. To date, many of these drugs have been used as inhibitors in the treatment of cancer and other diseases.

Kinase inhibitors are a group of molecules that specifically inhibit the action of one or more protein kinases. Hence, they can be subdivided or characterized by the amino acids whose phosphorylation is inhibited [3]. The general assumption is that they block the cell signaling function, but this is only part of the whole process. It is believed that their effect is dose dependent. When high doses of kinase inhibitors are administered to patients, it has been found that they prevent kinases from linking up with the Hsp90-Cdc37 chaperone system, a complex of molecules in cells that plays a vital role in the stability of proteins $[4,5]$. They have been heralded as a new type of targeted therapy, because there are 400 of them under development and there are 25 already in use. A few examples are erlotinib for the treatment of non-smallcell lung cancer, lapatinib for the treatment of HER2-positive breast cancer, and sorafenib for the treatment of kidney cancer.

A second class of SMDs are the proteasome inhibitors (PIs). Interestingly, transformed cells display greater susceptibility to this kind of drug than healthy tissues [6,7]. Lactacystin was the first

*Corresponding author: Ioannis Papasotiriou, Research Genetic Cancer Centre Ltd, M. Alexandrou 115 Str, 53070, Filotas, Greece, Tel: +30-24630-42264; Fax +30-24630-42265, E-mail: papasotiriou.ioannis@rgcc-genlab.com

Received January 28, 2014; Accepted April 14, 2014; Published April 28, 2014

Citation: Toloudi M, Apostolou P, Chatziioannou M, Kourtidou E, Vlachou I, et al. (2014) Recent Developments in Cancer Treatment: A Review. Pharmaceut Reg Affairs S1: 001. doi:10.4172/2167-7689.S1-001

Copyright: $\odot 2014$ Toloudi M, et al. This is an open-access article distributed under the terms of the Creative Commons Attribution License, which permits unrestricted use, distribution, and reproduction in any medium, provided the original author and source are credited. 
non-peptidic PIs. As SMDs have been proven to be more effective in multiple myeloma than other cancer patients, many of them have been introduced into clinical practice; as monotherapy or in combination with other already established compounds (carfilzomib, marizomib, and MLN9708) [8].

The urgent need for new targeted anticancer drugs has led to the development of an additional type of inhibitor known as cyclindependent kinase (CDK) inhibitors [9]. They function by arresting cells in the G1 phase of the cell cycle. Palbociclib is a US Food and Drug Administration (FDA)-approved investigational new drug, which is an oral and selective inhibitor of CDK 4 and 6. CDK 4 and 6 are two closely related kinases that enable tumor cell progression during the G1 and $\mathrm{S}$ phases of the cell cycle. Many broad-range CDK inhibitors such as flavopiridol and olomoucine, as well as specific CDK inhibitors such as fascaplysin, ryuvidine, and purvalanol A) are under investigation in clinical trials [10].

Generally, this type of molecule that can inhibit multiple targets simultaneously, including cell receptors or signal transduction pathway proteins, has been found to be less specific than therapeutic monoclonal antibodies. Consequently, the use of some of these molecules can lead to a higher risk of toxicity and gastrointestinal symptoms. Indeed, it has been shown that molecular-targeted therapies which are used in combination with conventional cytotoxic chemotherapy or moabs achieve improved outcomes [11].

\section{Moabs}

Because of their epitope-specific interactions, moabs still remain one of the most promising treatment strategies for cancer. They have the ability to target specific antigens such as hematopoietic differentiation antigens, extracellular matrix antigens, glycoproteins from solid tumors, molecules required for growth and differentiation, angiogenic inhibitors, and glycolipids and carbohydrates [12,13].

In accordance with their basic characteristics, moabs simply recognize the cancer cells and make them visible to the immune system. Ofatumumab (HuMax-CD20), a fully human B-cell-depleting agent, is a moab that was developed against the CD20 protein, which is primarily found on the surface of immune system B cells. Rituximab (Mabthera) used for the treatment of non-Hodgkin's lymphoma and alemtuzumab (MabCampath) are also compounds that belong to the same category [14-16].

Another group of moabs stimulate an anticancer immune response by binding to receptors on the surface of immune cells, and inhibiting signals that prevent them from attacking the cancer cells. Panitumumab (IgG2) and cetuximab (IgG1) are a type of moab that target the epidermal growth factor receptor (EGFR), although they differ in their isotype and thus in their mechanism of action. They may also trigger apoptosis and activate the immune system to destroy tumor cells $[17,18]$

Many interesting clinical aspects of moabs are discussed in this review. Thousands of compounds have already been approved by the US FDA, and many others are in clinical trials. Over the last few decades, a new generation of monoclonal-based drugs has been developed using Fc part modifications and phage display methods, as well as EpsteinBarr virus transformation procedures $[19,20]$. Despite the advantages offered by these drugs as mentioned above, patients still experience side effects that include flu-like symptoms, severe allergic reactions, organ damage (usually involving the heart, lungs, kidneys, liver, or brain), and others. Furthermore, their average affinity is generally lower than that of polyclonal antibodies. Concerning their specificity, moabs directed against conformational epitopes on native proteins may lose reactivity with antigens that have been minimally perturbed. Thus, they may sometimes display unexpected cross reactions with unrelated antigens.

Recently, researchers have discovered a unique monoclonal antibody that may effectively penetrate cancer cells, a key goal for these important anticancer agents, because most proteins that are associated with cancer are located inside the cell [21].

\section{ADCs}

The ADCs that are also called immunoconjugates belong to the moab family. The difference between moabs and ADCs is that the ADC molecule consists of an antibody that is linked with a small compound such as a plant or bacterial toxin, a chemotherapy drug, or a radioactive molecule. The mechanism of action is based on the fact that the moab drives the toxin into the cancer cell where it will manifest its cytotoxic activity. In fact, the process is more complex. When the ADC travels into the circulation, the linker that conjugates the moab with the toxin must be stable in order not to be damaged. However, the moab must have the ability to bind specifically to the target and be internalized by the cell to facilitate improved drug release (in an active form). Finally, the high efficacy of the ADC against the selected target is because of its toxicity [22]. 90Y-ibritumomab tiuxetan, which is an FDA-approved ADC, targets the CD20 antigen to deliver radioactive yttrium-90 to B-cell non-Hodgkin lymphoma cells. Another ADC is 131I-tositumomab, which targets the CD20 antigen to deliver radioactive iodine- 131 to non-Hodgkin lymphoma cells. MLN0264 is an experimental ADC designed to selectively deliver a highly potent cytotoxic payload to tumors that express guanyl cyclase C (GCC). MLN0264 consists of the highly toxic agent monomethyl auristatin E (MMAE) attached to an antibody that recognizes GCC via a cleavable linker [23].

In the case of ADCs, there are some disadvantages that still have to be overcome. For ADCs to be used in cancer treatment, the tumor needs to be tested for the expression of the target antigen. In addition, the payload of the ADC may become toxic as a result of premature release. Additionally, as was mentioned above, toxicity may be produced as a consequence of non-specific binding to healthy tissues and heterogeneous solid tumors [24].

\section{Cancer treatment vaccines}

Cancer treatment vaccines belong to the family of biological response modifiers. They are categorized as preventive (or prophylactic) vaccines and treatment (or therapeutic) vaccines. In the field of cancer, therapeutic vaccines are used that are either designed to treat cancers that have already developed or to prevent the disease.

With regard to the immune system's mode of action, it is capable of both specific and non-specific responses against tumor cells. For a cancer vaccine to be successful, it must have the ability to stimulate the immune system to act primarily in a tumor-specific fashion. In this mode, it would present tumor antigens to immune cells and activate CD4 (also known as helper T cells) and CD8 T cells (also known as cytotoxic or killer $\mathrm{T}$ cells). CD8 $\mathrm{T}$ cells when activated directly kill tumor cells [25], while CD4 T cells are indirectly activated by dendritic cells and macrophages [26] to produce messengers (cytokines) that boost CD8 (killer) T-cell activity $[27,28]$.

A variety of candidate cancer vaccines have shown promise in early (phase I and II) clinical studies. These vaccines are directed against renal cell carcinoma, melanoma, colon, breast, and prostate cancer, as well as blood (hematological) cancer. Almost all of them have progressed to phase III clinical studies. Sipuleucel-T (APC8015, trade 
name Provenge) is the first FDA-approved therapeutic cancer vaccine for prostate cancer. This is a dendritic cell vaccine; other types of cancer vaccine that are being studied are tumor cell vaccines, antigen vaccines, and DNA and vector-based vaccines $[29,30]$.

At this time point it has to be mentioned that this type of immunotherapy has systemic and local effects. Recent studies have shown that vaccination with embryonic stem cells may generate effective antitumor immunity against ovarian cancer [31]. Because whole cells are used for the preparation of vaccines, a greater proportion of intracellular molecules are combined with a smaller proportion of cell surface-associated molecules, thereby reducing the concentration of cancer cell-specific antigens recognized for vaccine production. Therefore, cancer cells used for the development of cancer treatment vaccines contain a high proportion of targets that may not be cancer cell specific, and an enrichment of cell surface material is needed to improve their effectiveness [32].

\section{Other Recent Treatments}

The development of nanopolymer drug delivery systems is another type of treatment for cancer. Liposomal and lipid-based products have entered the mainstream as sustained-release drug delivery systems. Marqibo (Talon) with the generic name vincristine sulfate is used for the treatment of patients with acute lymphoblastic leukemia (ALL); Myocet (Cephalon), a liposome - encapsulated form of doxorubicin ,is used for the treatment of breast cancer. These are two examples of the many FDA-approved drugs in this category $[33,34]$. Additionally, treatment methods that include combinations of therapeutic models already in use, have been investigated.

\section{Conclusions}

Among the most common cancer therapies such as chemotherapy, radiation therapy and surgery, the above mentioned treatment approaches are tools to prognose, diagnose, or treat cancer. The outcome of some of them are product which are in development or have been licensed by the FDA to be used in clinical practise. In conclusion, although such new generation of cancer treatment is associated with several adverse effects, such as acneiform rash, cardiac dysfunction, thrombosis, hypertension, and proteinuria, these targeted therapies (immunophenotyping, tumor genomics) were proven to generally be better tolerated than traditional chemotherapy. They have also proven to reduce the overall survival and improve the standard of living of cancer patients.

\section{References}

1. Hajdu SI (2011) A note from history: landmarks in history of cancer, part 1. Cancer 117: 1097-1102.

2. Salaverry O (2013) Cancer etymology and its historical curious course. Rev Peru Med Exp Salud Publica 30: 137-141.

3. Zhang J, Yang PL, Gray NS (2009) Targeting cancer with small molecule kinase inhibitors. Nat Rev Cancer 9: 28-39.

4. Polier S, Samant RS, Clarke PA, Workman P, Prodromou C, et al. (2013) ATPcompetitive inhibitors block protein kinase recruitment to the Hsp90-Cdc37 system. Nat Chem Biol 9: 307-312.

5. Vaughan CK, Mollapour M, Smith JR, Truman A, Hu B, et al. (2008) Hsp90dependent activation of protein kinases is regulated by chaperone-targeted dephosphorylation of Cdc37. Mol Cell 31: 886-895.

6. Voorhees PM, Orlowski RZ (2006) The proteasome and proteasome inhibitors in cancer therapy. Annu Rev Pharmacol Toxicol 46: 189-213.

7. Voorhees PM, Dees EC, O'Neil B, Orlowski RZ (2003) The proteasome as a target for cancer therapy. Clin Cancer Res 9: 6316-6325.
8. Moreau P, Richardson PG, Cavo M, Orlowski RZ, San Miguel JF, et al. (2012) Proteasome inhibitors in multiple myeloma: 10 years later. Blood 120: 947-959.

9. Krystof V, Uldrijan S (2010) Cyclin-dependent kinase inhibitors as anticancer drugs. Curr Drug Targets 11: 291-302.

10. Cicenas J, Valius M (2011) The CDK inhibitors in cancer research and therapy. J Cancer Res Clin Oncol 137: 1409-1418.

11. Widakowich C, de Castro G, Jr, de Azambuja E, Dinh P, Awada A (2007) Review: side effects of approved molecular targeted therapies in solid cancers. Oncologist 12:1443-1455.

12. Silacci M, Brack SS, Spath N, Buck A, Hillinger S, et al. (2006) Human monoclonal antibodies to domain $\mathrm{C}$ of tenascin-C selectively target solid tumors in vivo. Protein Eng Des Sel 19: 471-478. http://www.ncbi.nlm.nih.gov/entrez/ query.fcgi?cmd=Retrieve\&db=PubMed\&dopt=Citation\&list_uids=16928692

13. Murakami H, Doi T, Yamamoto N, Watanabe J, Boku N, et al. (2012) Phase 1 study of ganitumab (AMG 479), a fully human monoclonal antibody against the insulin-like growth factor receptor type I (IGF1R), in Japanese patients with advanced solid tumors. Cancer Chemother Pharmacol 70: 407-414.

14. Jain P, O'Brien S (2013) Anti-CD20 monoclonal antibodies in chronic lymphocytic leukemia. Expert Opin Biol Ther 13: 169-182.

15. Golay J, Da Roit F, Bologna L, Ferrara C, Leusen JH, et al. (2013) Glycoengineered CD20 antibody obinutuzumab activates neutrophils and mediates phagocytosis through CD16B more efficiently than rituximab. Blood 122: 3482-3491.

16. Bhutani D, Vaishampayan UN (2013) Monoclonal antibodies in oncology therapeutics: present and future indications. Expert Opin Biol Ther 13: 269-282.

17. Park NJ, Wang X, Diaz A, Goos-Root DM, Bock C, et al. (2013) Measurement of cetuximab and panitumumab-unbound serum EGFR extracellular domain using an assay based on slow off-rate modified aptamer (SOMAmer) reagents. PLoS One 8.

18. Yewale C, Baradia D, Vhora I, Patil S, Misra A (2013) Epidermal growth factor receptor targeting in cancer: a review of trends and strategies. Biomaterials 34: 8690-8707.

19. Karpas A, Dremucheva A, Czepulkowski BH (2001) A human myeloma cell line suitable for the generation of human monoclonal antibodies. Proc Natl Acad Sci U S A 98: 1799-1804.

20. Scott AM, Allison JP, Wolchok JD (2012) Monoclonal antibodies in cancer therapy. Cancer Immun 12: 14.

21. Veomett N, Dao T, Scheinberg DA (2013) Therapeutic antibodies to intracellular targets in cancer therapy. Expert Opin Biol Ther 13:1485-1488.

22. Ducry L, Stump B (2010) Antibody-drug conjugates: linking cytotoxic payloads to monoclonal antibodies. Bioconjug Chem 21: 5-13.

23. (2013) Antibody-drug conjugate may inhibit pancreatic cancer. Cancer Discov 3.

24. Teicher BA, Chari RV (2011) Antibody conjugate therapeutics: challenges and potential. Clin Cancer Res 17: 6389-6397.

25. Janeway CA Jr, Bottomly K (1994) Signals and signs for lymphocyte responses Cell 76: 275-285.

26. Grohmann U, Fioretti MC, Bianchi R, Belladonna ML, Ayroldi E, et al. (1998) Dendritic cells, interleukin 12, and CD4+ lymphocytes in the initiation of class I-restricted reactivity to a tumor/self peptide. Crit Rev Immunol 18: 87-98.

27. Seder RA, Paul WE (1994) Acquisition of lymphokine-producing phenotype by CD4+ T cells. Annu Rev Immunol 12: 635-673.

28. Harrop R (2013) Cancer vaccines: identification of biomarkers predictive of clinical efficacy. Hum Vaccin Immunother 9: 800-804.

29. Fiorito C, Gontero P (2013) Adverse events after intravesical therapy: are we always ready to recognize and prevent them? Urologia 21: 27-32.

30. Zhang Z, Chen X, Chang X, Ye X, Li Y, et al. (2013) Vaccination with embryonic stem cells generates effective antitumor immunity against ovarian cancer. Int $J$ Mol Med 31: 147-153.

31. Lokhov PG, Balashova EE (2010) Cellular cancer vaccines: an update on the development of vaccines generated from cell surface antigens. J Cancer 1: 230-241.

32. Allen TM, Cullis PR (2004) Drug delivery systems: entering the mainstream Science 303: 1818-1822 
Citation: Toloudi M, Apostolou P, Chatziioannou M, Kourtidou E, Vlachou I, et al. (2014) Recent Developments in Cancer Treatment: A Review. Pharmaceut Reg Affairs S1: 001. doi:10.4172/2167-7689.S1-001

33. Medina OP, Zhu Y, Kairemo K (2004) Targeted liposomal drug delivery in cancer. Curr Pharm Des 10: 2981-2989.

34. Wang Y, Minko T (2004) A novel cancer therapy: combined liposomal hypoxia inducible factor 1 alpha antisense oligonucleotides and an anticancer drug. Biochem Pharmacol 68: 2031-2042.

This article was originally published in a special issue, New Therapeutic

Approaches in Treating Cancer handled by Editor(s). Prof. Fabio Grizzi,

Humanitas Clinical and Research Center, Italy 